\title{
Assignments in the electronic spectrum of water
}

\author{
Danny Yeager and Vincent McKoy ${ }^{\dagger}$ \\ Arthur Amos Noyes Laboratory of Chemical Physics, ${ }^{*}$ California Institute of Technology, Pasadena, California \\ 91109
}

\section{G. A. Segal ${ }^{\dagger}$}

Department of Chemistry, University of Southern California, Los Angeles, California 90024

(Received 3 October 1973)

To explain the inelastic feature at $4.5 \mathrm{eV}$ in the spectrum of water and to study its spectrum in some detail, we have carried out several calculations on the excited states of water using the equations-of-motion method. We conclude that the calculated vertical excitation energy of $6.9 \mathrm{eV}$ for the ${ }^{3} B_{1}$ state corresponds to the strong feature at $7.2 \mathrm{eV}$ observed in low-energy electron scattering spectrum. The $4.5 \mathrm{eV}$ inelastic process almost certainly does not correspond to a vertical excitation of water at the ground state geometry. The other excitation energies and oscillator strengths agree well with experiment.

\section{INTRODUCTION}

Despite the importance of the water molecule there is considerable question as to the location, intensities, and nature of transitions to its lower excited electronic states. A recent study by Claydon, Segal, and Taylor ${ }^{1}$ which combined available experimental evidence with the suggestions of semiempirical calculations provided a consistent interpretation of the facts known at the time. Even more recently, however, Knoop, Brongersma, and Oosterhoff ${ }^{2}$ and Trajmar et al. ${ }^{3}$ have reported electron impact spectra of $\mathrm{H}_{2} \mathrm{O}$ which raise a serious question as to the nature of the inelastic process observed by many workers at $4.5 \mathrm{eV}$ in $\mathrm{H}_{2} \mathrm{O}$. Despite the fact that the calculation of the excitation energy to the lowest triplet state of a molecule should be straightforward, the calculations reported in the literature ${ }^{1}$ all find the ${ }^{3} B_{1}$ state to be the lowest excited state of $\mathrm{H}_{2} \mathrm{O}$ and to have a vertical excitation energy above $6 \mathrm{eV}$. Moreover there are no reliable or extensive calculations of the oscillator strengths for the several dipole-allowed transitions below $10 \mathrm{eV}$. These quantities are needed in the analysis of the relative intensities of several transitions observed in the electron impact spectrum of water."

In view of this discrepancy and the questions raised by recent electron impact spectra of $\mathrm{H}_{2} \mathrm{O}^{2,3}$ and in order to provide reliable estimates of the oscillator strengths of several transitions, we have carried out an extensive calculation of the excited state manifold of $\mathrm{H}_{2} \mathrm{O}$ at the vertical geometry using the equations-of-motion method. ${ }^{5}$ One of our conclusions from this study is that the vertical excitation energy to the ${ }^{3} B_{1}$ state is in the vicinity of $6.9 \mathrm{eV}$ and almost certainly corresponds to the strong feature at $7.2 \mathrm{eV}$ observed by Knoop et al. ${ }^{2}$ in their low-energy electron scattering spectrum. The triplet character of the feature at $7.2 \mathrm{eV}$ is also supported by the electron impact spectra of Trajmar et al ${ }^{3,6}$ The $4.5 \mathrm{eV}$ inelastic process almost certainly does not correspond to a vertical excitation of $\mathrm{H}_{2} \mathrm{O}$ in the ground state geometry. The experimental evidence regarding the existence of an inelastic feature at $4.5 \mathrm{eV}$ seems unambiguous $^{6}$ and to put this problem into perspective we shall begin by considering the experimental evidence regarding the 4.5 and $7.2-7.5 \mathrm{eV}$ regions. We shall then describe our calculations of the excitation energies and $f$ values of several transitions by the equations-ofmotion method. The results, including the $f$ values, agree well with available data.

\section{EXPERIMENTAL EVIDENCE FOR STATES AT 4.5 AND $7.2-7.5 \mathrm{eV}$}

Until recent low-energy electron impact spectra were reported ${ }^{2,3,6}$ there was a real question as to the nature and existence of the inelastic feature at $4.5 \mathrm{eV}$ in the spectrum of water. The feature is extremely weak and has never been observed in the gas phase optical spectrum. However, Larzal et al ${ }^{7}$ observed weak absorption at $4.5 \mathrm{eV}$ using an $80 \mathrm{~cm}$ path length of liquid water. This process presumably corresponds to the structure at $4.0 \pm 0.1 \mathrm{eV}$ reported by Hunter, Lewis, and Hamill ${ }^{8}$ in their low-energy electron reflection spectra of a thin film of ice at $77^{\circ} \mathrm{K}$. These authors $\mathrm{s}^{7,8}$ attribute their observation to absorption to the ${ }^{3} B_{1}$ state of water, presumably in the Franck-Condon region. No structure was observed at lower energy. ${ }^{7,8}$ This absorption observed in condensed phases could conceivably be due to a number of causes other than excitation to an excited state of $\mathrm{H}_{2} \mathrm{O}$. In particular, dimer absorption or absorption due to $\mathrm{OH}$ radicals formed through radiation damage are possible, although the dissociation energy for the process $\mathrm{H}_{2} \mathrm{O} \rightarrow \mathrm{H}\left({ }^{2} S\right)+\mathrm{OH}\left(X^{2} \mathrm{II}\right)$ is $5.11 \mathrm{eV}$.

Hamill and co-workers ${ }^{9,10}$ have, however, provided additional, but indirect and inconclusive evidence for the existence of a state around 4.5 eV. By electron impact on alcohols a water fragment can be formed in an excited state. For example, for ethanol,

$$
e^{-}+\mathrm{C}_{2} \mathrm{H}_{5} \mathrm{OH} \rightarrow \mathrm{C}_{2} \mathrm{H}_{4}^{+}+\mathrm{H}_{2} \mathrm{O}+2 e^{-} \text {. }
$$

The ionization efficiency curve for $\mathrm{C}_{2} \mathrm{H}_{4}^{+}$from ethanol exhibited several "breaks" which occur at the same energy intervals as those for $\mathrm{C}_{2} \mathrm{H}_{4}^{+}$from ethane and ethylene, but $4.3 \mathrm{eV}$ above the onset for this process there was an additional break in the efficiency curve. At the onset of the process, $\mathrm{H}_{2} \mathrm{O}$ in its ground state was formed, but at $4.3 \mathrm{eV}$ above onset excited state water can also 
be formed so that an upward break in the efficiency curve is produced by an additional channel for $\mathrm{C}_{2} \mathrm{H}_{4}^{+}$ generation. Lewis and Hamill ${ }^{11}$ have also reported that this process occurs for the cyclic alcohols $\mathrm{c}-\mathrm{C}_{5} \mathrm{H}_{9} \mathrm{OH}$ and $\mathrm{c}-\mathrm{C}_{6} \mathrm{H}_{11} \mathrm{OH}$ to produce water and $\mathrm{C}_{5} \mathrm{H}_{8}^{+}$and $\mathrm{C}_{6} \mathrm{H}_{10}^{+}$, respectively. By methods similar to those used for ethanol, Lewis and Hamill 11 found and extra "break" in the efficiency curves at 4.3 and $4.5 \mathrm{eV}$ for $\mathrm{c}-\mathrm{C}_{5} \mathrm{H}_{9} \mathrm{OH}$ and $\mathrm{c}-\mathrm{C}_{6} \mathrm{H}_{11} \mathrm{OH}$, respectively. These experiments offer supplementary evidence for a value of the lowest singlet-triplet excitation energy of about $4.5 \mathrm{eV}$. It is important to realize that in the case of $\mathrm{H}_{2} \mathrm{O}$ being formed as a neutral fragment of the electron impact on alcohols, the value measured might represent a minimum (or nonvertical) transition energy rather than a transition determined by Franck-Condon factors.

More definitive data for the existence of the inelastic feature at $4.5 \mathrm{eV}$ are provided by low-energy electron impact spectra since the cross section for excitation of triplet states is considerably enhanced under these conditions. These cross sections also have a distinctive angular distribution. The $4.5 \mathrm{eV}$ feature is weak in these spectra. Schul $\mathrm{z}^{12}$ observed an energy loss process with an onset of about $3.4 \mathrm{eV}$ with the trapped electron method. A threshold electron impact spectrum using $\mathrm{SF}_{6}$ as a scavenger ${ }^{13}$ was similar to Schulz's spectrum except that the low-energy electron loss feature was observed to have an onset at $4.4 \mathrm{eV}$. Raff ${ }^{14}$ observed the feature on electron impact with $30 \mathrm{eV}$ electrons while Lassettre ${ }^{15}$ did not observe this feature in the electron impact spectrum at low scattering angles and impact energies above $100 \mathrm{eV}$. However Lassettre ${ }^{16} \mathrm{did}$ observe very weak scattering with an onset of about 4.4 $\mathrm{eV}$ in another spectra at low scattering angles and impact energies between 30 and $60 \mathrm{eV} \cdot{ }^{16}$ From their trapped electron spectrum of water, Azria and FiquetFayard ${ }^{17}$ concluded that the $4.5 \mathrm{eV}$ energy loss feature is due to contamination, but the preponderance of evidence is clearly against this conclusion. From a study of the pressure and primary beam dependence of the trapped electron current Knopp has shown that it is very unlikely that the $4.5 \mathrm{eV}$ feature could be due to excitation of $\mathrm{OH}$ radicals or molecular complexes of water. ${ }^{18}$

More detailed and convincing data are provided by the work of Trajmar and co-workers. ${ }^{3,6}$ They have shown that the differential cross section of electrons inelastically scattered with an energy loss of $4.5 \mathrm{eV}$ is consistent with a singlet-tripl et transition. Their studies also rule out any contribution to this feature from water dimers or hot bands. In more recent spectra at very low impact energies Trajmar $^{6}$ has also observed the $4.5 \mathrm{eV}$ feature with a very enhanced cross section and also a transition at $7.2 \mathrm{eV}$ with the characteristics of a singlet-triplet transition. Finally, Knopp et al . ${ }^{2}$ recently reported a low-energy electron impact spectrum taken by the double retarding potential difference method. They observed the $4.5 \mathrm{eV}$ inelastic feature and, more interestingly, also found a strong transition peaked at $7.2 \mathrm{eV}$ which is distinct from the $7.5 \mathrm{eV}\left({ }^{1} B_{1}\right)$ feature and which they assign as due to the ${ }^{3} B_{1}$ or ${ }^{3} A_{2}$ state. They did not find a peak at $7.5 \mathrm{eV}$ for the cross section, for singlets are low in their method.

This is the current experimental situation on the transitions in $\mathrm{H}_{2} \mathrm{O}$ at 4.5 and also 7.2 and $7.5 \mathrm{eV}$. We now discuss our calculations on the spectra of the water molecule from which we will assign the transition at $7.2 \mathrm{eV}$ as the vertical excitation to the ${ }^{3} B_{1}$ state. Our results also show that there is no vertical excitation energy at the ground state geometry in this molecule around $4.5 \mathrm{eV}$.

\section{THEORY}

We have used the equations-of-motion method to calculate the excitation energies and oscillator strengths for several transitions in the water molecule. The equations-of-motion method is an approach for the direct calculation of the properties of physical interest in spectroscopy, e.g., excitation energies and intensities. By using a theory specifically designed for studying these relative properties one can avoid many of the difficulties involved in obtaining highly accurate values for absolute quantities such as the total energies. We have discussed this method in recent papers ${ }^{5,19}$ and here we will give only a very brief summary of the theory. It can be shown that the operator $O_{\lambda}^{+}$which generates an excited state $|\lambda\rangle$ from the ground state, i. e., $|\lambda\rangle$ $=O_{\lambda}^{+}|0\rangle$, is exactly a solution of the equation of motion, ${ }^{20}$

$$
\left\langle 0\left|\left[\delta O_{\lambda}, H, O_{\lambda}^{+}\right]\right| 0\right\rangle=\omega_{\lambda}\left\langle 0\left|\left[\delta O_{\lambda}, O_{\lambda}^{+}\right]\right| 0\right\rangle,
$$

where $\delta O_{\lambda}^{+}$is a variation of the amplitudes specifying $O_{\lambda}^{+}$and $\omega_{\lambda}$ the excitation frequency. The double commutator is defined as

$$
2[A, B, C]=[[A, B] C]+[A,[B, C]] \text {. }
$$

We have derived various approximations to the solution of Eq. (1). ${ }^{5,19}$ If $O_{\lambda}^{+}$is restricted to single particle-hole $(1 p-1 h)$ form, Eq. (1) becomes

$$
\left[\begin{array}{cc}
\mathbf{A} & \mathbf{B} \\
-\mathbf{B}^{*} & -\mathrm{A}^{*}
\end{array}\right]\left[\begin{array}{l}
\mathbf{Y}(\lambda) \\
\mathbf{Z}(\lambda)
\end{array}\right]=\omega_{\lambda}\left[\begin{array}{ll}
\mathbf{D} & O \\
O & \mathrm{D}
\end{array}\right]\left[\begin{array}{l}
\mathbf{Y}(\lambda) \\
\mathbf{Z}(\lambda)
\end{array}\right],
$$

where the elements of A, B, and D are defined in Ref. 5 and $Y(\lambda)$ and $\mathrm{Z}(\lambda)$ are the amplitudes of $O_{\lambda}^{+}$. We have also derived perturbationlike schemes for solving Eq. (1) including both $1 p-1 h$ and $2 p-2 h$ operators. ${ }^{19}$ At this level of approximation we have obtained accurate excitation energies and oscillator strengths for transitions in $\mathrm{N}_{2},{ }^{21} \mathrm{CO},{ }^{21} \mathrm{C}_{2} \mathrm{H}_{4},{ }^{21} \mathrm{H}_{2} \mathrm{CO},{ }^{22} \mathrm{CO}_{2},{ }^{23}$ and $\mathrm{C}_{6} \mathrm{H}_{6} .{ }^{24}$ We now discuss the application of this method to the spectrum of the water molecule.

\section{RESULTS}

The electron configuration of the ground state of water is

$$
\left(1 a_{1}\right)^{2}\left(2 a_{1}\right)^{2}\left(1 b_{2}\right)^{2}\left(3 a_{1}\right)^{2}\left(1 b_{1}\right)^{2} .
$$

In an equations-of-motion calculation one first carries out a self-consistent field calculation on the ground state of the molecule to generate a particle-hole basis. We have used the currently accepted ground state experimental geometry ${ }^{25}$ and carried out calculations ${ }^{26}$ using different basis sets to study the effect of the composi- 
tion of the orbital basis on the excitation energies and $f$ values. We will refer primarily to the results obtained in the largest basis but will also discuss the results in other basis sets whenever the differences in the results illustrate some important feature. For this largest basis we used a $[3 s 2 p / 1 s]$ valence basis contracted from a $(7 s 3 p / 3 s)$ Gaussian basis ${ }^{27}$ to which we added a diffuse $s$ function with an orbital exponent of 0.038 on each hydrogen and two $s$ functions with exponents of 0.089 and 0.022 and two sets of $p$ functions also with exponents of 0.089 and 0.022 on the oxygen center. Polarization functions have been shown to be important in ground state SCF calculations on water ${ }^{28}$ and hence we added to our basis a contracted set of $d$-polarization functions on oxygen with exponents of 1.322 and 0.3916 and coefficients 0.3579 and 0.7596 , respectively, and a set of $p$ polarization functions, on hydrogen with an exponent of 1.16. Another calculation was done with this same basis but from which the polarization functions were deleted. The differences in the results of the two calculations are not very significant but may amount to $0.3-0.5 \mathrm{eV}$ in the excitation energies to some states. In the calculations, all molecular orbital levels except the highest particle state and the lowest hole state are included. ${ }^{29}$

Table I shows the excitation energies for ten transitions in water along with the oscillator strengths for the four dipole-allowed transitions. These are results of the calculation using the $1 p-1 h$ plus $2 p-2 h(1 p-1 h+2 p-$ $2 h$ ) approximation ${ }^{19}$ to the solution of the equation of motion, Eq. (1), and the basis set containing the polarization functions. The lowest calculated vertical excitation energy is about $6.9 \mathrm{eV}$ and is to the ${ }^{3} B_{1}\left(1 b_{1}-3 s\right)$ state. The corresponding singlet transition energy is $7.2 \mathrm{eV}$ and hence the $B_{1}$ singlet-triplet splitting is $0.3 \mathrm{eV}$. Hence we can now assign the peak observed by Knopp ${ }^{2}$ at $7.2 \mathrm{eV}$ as the ${ }^{3} B_{1}$ state, $0.2 \mathrm{eV}$ below the observed

TABLE I. Vertical excitation energies and oscillator strengths in water. ${ }^{2}$

\begin{tabular}{cllllll}
\hline \hline State & $\begin{array}{l}\text { Main } \\
\text { transition }\end{array}$ & $\begin{array}{l}\Delta E^{\mathrm{b}} \\
\text { (EOM) }\end{array}$ & $\begin{array}{l}\Delta E \\
\text { (Obs.) }\end{array}$ & $\begin{array}{l}\Delta E^{\mathrm{c}} \\
(\mathrm{CI})\end{array}$ & $\begin{array}{l}f^{\mathrm{b}} \\
\text { (EOM) }\end{array}$ & $\begin{array}{l}f \\
\text { (Obs.) }\end{array}$ \\
\hline$\tilde{A}^{3} B_{1}$ & $1 b_{1} \rightarrow 3 s$ & 6.89 & $7.2^{\mathrm{d}}$ & 7.3 & & \\
$\tilde{A}^{1} B_{1}$ & $1 b_{1} \rightarrow 3 s$ & 7.22 & $7.4^{\mathrm{e}}$ & 7.6 & 0.05 & $0.04^{\mathrm{f}}$ \\
${ }^{3} A_{2}$ & $1 b_{1} \rightarrow 3 p y$ & 8.97 & & 9.3 & & \\
${ }^{1} A_{2}$ & $1 b_{1} \rightarrow 3 p y$ & 9.02 & $9.1^{\mathrm{d}}$ & 9.5 & & \\
${ }^{3} A_{1}$ & $3 a_{1} \rightarrow 3 s$ & 9.34 & & 9.4 & & \\
${ }^{3} A_{1}$ & $1 b_{1} \rightarrow 3 p x$ & 9.39 & & & & \\
${ }^{3} B_{1}$ & $1 b_{1} \rightarrow 3 p z$ & 9.47 & & & & \\
$\tilde{C}^{1} B_{1}$ & $1 b_{1} \rightarrow 3 p z$ & 9.48 & $10.0^{\mathrm{g}}$ & & 0.006 & $\ldots . \mathrm{h}$ \\
$\widetilde{B}^{1} A_{1}$ & $3 a_{1} \rightarrow 3 s$ & 9.54 & 9.67 & 9.8 & 0.06 & $0.05^{\mathrm{g}}$ \\
$\tilde{D}^{1} A_{1}$ & $1 b_{1} \rightarrow 3 p x$ & 9.61 & 10.17 & & 0.02 & $\cdots . \mathrm{h}$ \\
\hline \hline
\end{tabular}

${ }^{a}$ All energies in electron volts.

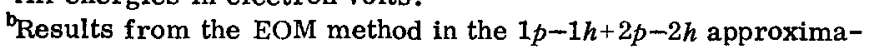
tion. See Ref. 19.

${ }^{c} N$. W. Winter (private communication).

${ }^{d}$ Reference 2.

'Reference 3 .

${ }^{\mathrm{f}} \mathrm{K}$. Watanabe and M. Zelikoff, J. Opt. Soc. Am. 43, 753 (1953).

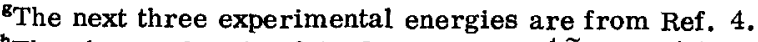

The observed ratio of $f$ values for the $D^{1} \tilde{A}_{1}$ and $C^{1} \widetilde{B}_{1}$ states is about 1.2 (see Ref. 4). transition to the ${ }^{1} B_{1}$ state. ${ }^{3}$ Clearly the inelastic feature at $4.5 \mathrm{eV}$ is not a vertical transition to the ${ }^{3} B_{1}$ state. From the accuracy of the results on several other molecules we are confident that the predicted value of 6.9 $\mathrm{eV}$ for the vertical excitation energy to the ${ }^{3} B_{1}$ state would be within $5 \%-10 \%$ of the true value. If one assumes that the transition is highly nonvertical the ${ }^{3} B_{1}$ potential energy curve would have to drop by over $2 \mathrm{eV}$ relative to the vertical value. Bader and Gangi ${ }^{30}$ calculated the energy difference between linear $\mathrm{H}_{2} \mathrm{O}$ and $\mathrm{H}_{2} \mathrm{O}$ in the ground state geometry to be $0.13 \mathrm{eV}$ for the loweșt triplet surface. Linear $\mathrm{H}_{2} \mathrm{O}$ is only a saddle point on this surface. The calculated $f$ value of 0.05 for the transition to the $\tilde{A}^{1} B_{1}$ state agrees well with the experimental value of 0.04 .

We assign the peak observed at $9.1 \mathrm{eV}$ by Knopp et al ${ }^{2}$ as a transition to the ${ }^{1} A_{2}\left(1 b_{1} \rightarrow 3 p y\right)$ state with a calculated excitation energy of $9.02 \mathrm{eV}$. The $\widetilde{B}{ }^{1} A_{1}\left(3 a_{1}-3 \mathrm{~s}\right)$ excitation energy of $9.54 \mathrm{eV}$ agrees well with the observed value of 9.67 . The triplet state observed by Trajmar $^{3}$ at $9.81 \mathrm{eV}$ is probably a transition to one of three triplet states, i. e., the ${ }^{3} A_{1}\left(3 a_{1}-3 s\right)$ at $9.34 \mathrm{eV}$, ${ }^{3} A_{1}\left(1 b_{1}-3 p x\right)$ at $9.39 \mathrm{eV}$, or the ${ }^{3} B_{1}\left(1 b_{1} \rightarrow 3 p z\right)$ at 9.47 $\mathrm{eV}$. From the good agreement between the calculated and observed excitation energies for the ${ }^{1} A_{1}\left(3 a_{1} \rightarrow 3 s\right)$ state, the assignment may be narrowed down to the ${ }^{3} A_{1}\left(1 b_{1}-3 p x\right)$ or ${ }^{3} B_{1}\left(1 b_{1}-3 p z\right)$ states. The $\tilde{B}^{1} A_{1}$ excitation energy of $9.54 \mathrm{eV}$ agrees well with the observed value of $9.67 \mathrm{eV}$ while the excitation energies to the $\tilde{\mathrm{C}}^{1} B_{1}$ $\left(1 b_{1}-3 p z\right)$ and $D^{1} A_{1}\left(1 b_{1}-3 p x\right)$ states are both about $5 \%$ below the observed values. The predicted $f$ value of 0.06 for the transition to the $\tilde{B}^{1} A_{1}$ state, observed as a broad continuum, is close to the experimental value of 0.05 . There are no experimental $f$ values available for transitions to the $\tilde{D}^{1} A_{1}$ and $\tilde{C}{ }^{1} B_{1}$ states, but Lassettre ${ }^{4}$ estimated the ratio of $f$ values for these two transitions to be about 1.3 from high energy electron impact studies. Our calculated $f$ values give a ratio of about 3 .

As expected, the excitation energies obtained from the calculations without the polarization functions are very close to those in Table I for transitions to states which are primarily excitations out of the $1 b_{1}$ orbital. This is because the $1 b_{1}$ orbital is almost the oxygen $2 p x$ orbital which is relatively nonbonding. However the excitation energies to states which arise primarily from an excitation out of the $3 a_{1}$ bonding orbital are all about $0.3-$ $0.4 \mathrm{eV}$ lower than those of Table I.

\section{CONCLUSIONS}

The available experimental evidence for the existence of an inelastic feature at $4.5 \mathrm{eV}$ in the spectrum of water is very convincing. To explain the nature of this process and to study the spectrum of water in some detail we have carried out several calculations on the excited states of water at the vertical geometry using the equations-of-motion method. ${ }^{5}$ We conclude that our calculated vertical excitation energy of $6.9 \mathrm{eV}$ for the ${ }^{3} B_{1}$ state corresponds to the strong feature at $7.2 \mathrm{eV}$ observed by Knoop et al. ${ }^{2}$ in their low-energy electron scattering spectrum. The $4.5 \mathrm{eV}$ inelastic process almost certainly does not correspond to a vertical excitation of 
water at the ground state geometry. The other excitation energies and oscillator strengths agree well with experiment. We can assign the state observed at 9.1 $\mathrm{eV}^{2}$ as a ${ }^{1} A_{2}\left(1 b_{1}-3 p y\right)$ and the triplet state at $9.81 \mathrm{eV}$ as probably a ${ }^{3} A_{1}$ or ${ }^{3} B_{1}$ and not a ${ }^{3} A_{2}$ excitation.

*Contribution No. 4768 .

tAlfred P. Sloan Foundation Fellow.

${ }^{1}$ C. R. Claydon, G. A. Segal, and H. S. Taylor, J. Chem. Phys. 54, 3799 (1971).

${ }^{2}$ F. W. E. Knopp, H. H. Brongersma, and C. J. Oosterhoff, Chem. Phys. Lett. 13, 20 (1972).

${ }^{3}$ S. Trajmar, W. Williams, and A. Kuppermann, J. Chem. Phys. 58, 2521 (1973).

${ }^{4}$ A. Skerbele, V. D. Meyer, and E. N. Lassettre, J. Chem. Phys. 43, 817 (1965).

${ }^{5}$ T. Shibuya and V. McKoy, Phys. Rev. A 2, 2208 (1970).

${ }^{6} \mathrm{~S}$. Trajmar (private communication).

${ }^{7}$ H. Larzal, F. Gelebart, and A. Johannin-Gilles, Compt. Rend. 261, 4701 (1965).

${ }^{8}$ L. M. Hunter, D. Lewis, and W. H. Hamill, J. Chem. Phys. 52, $1733(1970)$.

${ }^{9}$ D. Lewis and W. H. Hamill, J. Chem. Phys. 51, 456 (1969).

${ }^{10}$ C. E. Melton and W. H. Hamill, J. Chem. Phys 41, 546 (1964).

${ }^{11}$ D. Lewis and W. H. Hamill, J. Chem. Phys. 52, 6348 (1970).

${ }^{12}$ G. J. Schulz, J. Chem. Phys. 33, 1661 (1960).

${ }^{13}$ R. N. Compton, R. H. Huebner, P. W. Reinhardt, and L. G. Christophorou, J. Chem. Phys. 48, 901 (1968).

${ }^{14}$ L. M. Raff, PH. D. thesis, University of Illinois, Urbana, IL, 1962.

${ }^{15}$ (a)E. N. Lassettre and S. A. Francis, J. Chem. Phys. 40, 1208 (1964); (b) A. Skerbele and E. N. Lassettre, J. Chem. Phys. 42, 395 (1965); (c) E. N. Lassettre, A. Skerbele, M. A. Dillion, and K. J. Ross, J. Chem. Phys. 48, 5066
(1968).

${ }^{16}$ A. Skerbele, M. A. Dillon, and E. N. Lassettre, J. Chem. Phys. 49, 5042 (1968).

${ }^{17}$ R. Azria and F. Fiquet-Fayard, Compt. Rend. B273, 944 (1971).

${ }^{18}$ F. W. E. Knopp, Ph.D. thesis, Rijks University, Lerden, The Netherlands, 1972.

${ }^{19}$ T. Shibuya, J. Rose, and V. McKoy, J. Chem. Phys. 58, 500 (1973).

${ }^{20}$ D. J. Rowe, Rev. Mod. Phys. 40, 153 (1968).

${ }^{21}$ J. Rose, T. Shibuya, and V. McKoy, J. Chem. Phys. 58, 74 (1973).

${ }^{22}$ D. L. Yeager and V. McKoy, "The equations-of-motion method: Excitation energies and intensities in formaldehyde," J. Chem. Phys. (to be published).

${ }^{23} \mathrm{C}$. W. McCurdy and V. McKoy, "Differential cross sections for the scattering of electrons by $\mathrm{CO}_{2}$," J. Chem. Phys. (to be published).

${ }^{24} \mathrm{~J}$. Rose, T. Shibuya, and V. McKoy, "Electronic excitations of benzene from the equations-of-motion method," $J$. Chem. Phys. (to be published).

${ }^{25}$ W. S. Benedict, N. Gailar, and E. H. Plyler, J. Chem. Phys 24, 1139 (1956).

${ }^{26}$ The molecule is in the $y z$ plane with the corrdinate system centered on the oxygen and the hydrogens are in the first and second quadrants of the plane.

${ }^{27}$ For details of this basis see W. Coughran, J. Rose, T. Shibuya, and V. McKoy, J. Chem. Phys. 58, 2699 (1973).

${ }^{28}$ T. H. Dunning, Jr., J. Chem. Phys. 55, 3958 (1971).

${ }^{29}$ We have found that truncation of the particle spectrum can lead to a poor description of correlation effects in the excitation and that the location of the origin of the diffuse functions can be important for a small molecule such as $\mathrm{H}_{2} \mathrm{O}$. For larger molecules such as $\mathrm{H}_{2} \mathrm{CO}$ these considerations were not as important.

${ }^{30}$ R. W. Bader and R. A. Gangi, Chem. Phys. Lett. 6, 312 (1970). 